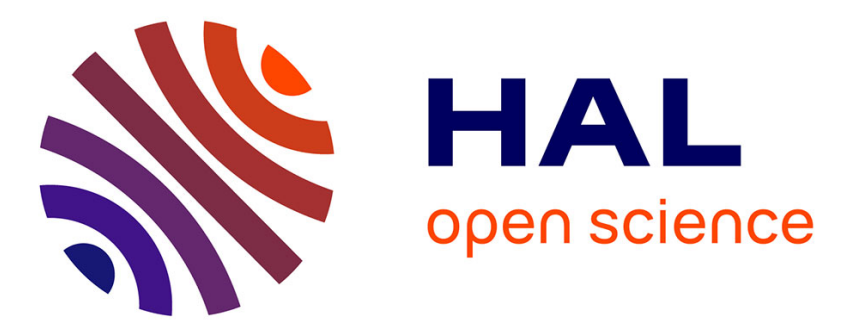

\title{
Deuteron magnetic resonance in liquid crystals : the interplay of molecular ordering and conformational changes
}

B. Deloche, J. Charvolin

\section{- To cite this version:}

B. Deloche, J. Charvolin. Deuteron magnetic resonance in liquid crystals: the interplay of molecular ordering and conformational changes. Journal de Physique Lettres, 1980, 41 (2), pp.39-42. 10.1051/jphyslet:0198000410203900 . jpa-00231716

\section{HAL Id: jpa-00231716 https://hal.science/jpa-00231716}

Submitted on 1 Jan 1980

HAL is a multi-disciplinary open access archive for the deposit and dissemination of scientific research documents, whether they are published or not. The documents may come from teaching and research institutions in France or abroad, or from public or private research centers.
L'archive ouverte pluridisciplinaire HAL, est destinée au dépôt et à la diffusion de documents scientifiques de niveau recherche, publiés ou non, émanant des établissements d'enseignement et de recherche français ou étrangers, des laboratoires publics ou privés. 


\title{
Deuteron magnetic resonance in liquid crystals : the interplay of molecular ordering and conformational changes $(*)$
}

\author{
B. Deloche and J. Charvolin \\ Laboratoire de Physique des Solides (**), Université de Paris-Sud, 91405 Orsay, France
}

(Reçu le 15 novembre 1979, accepté le 29 novembre 1979)

\begin{abstract}
Résumé. - Un examen des résultats de résonance magnétique des deutérons dans différents systèmes thermotropes remet en question l'interprétation récemment proposée par Bos et Doane suivant laquelle (i) la conformation moléculaire moyenne ne change pas avec la température, même aux transitions de phase, et (ii) les mésophases uniaxiales et biaxiales $\left(\mathrm{Sm}_{\mathrm{c}}\right)$ sont respectivement caractérisées par deux et trois paramètres d'ordre. Quelques contre-exemples indiquent que ces points ne sont pas généraux et, dans certains cas, ambigus. Le rôle des changements conformationnels ne peut être négligé.
\end{abstract}

\begin{abstract}
Examination of deuteron magnetic resonance data in different thermotropic systems calls into question the explanation recently proposed by Bos and Doane wherein (i) the average molecular conformation does not change with temperature, even at phase transitions, and (ii) uniaxial and biaxial $\left(\mathrm{Sm}_{\mathrm{c}}\right)$ mesophases are characterized by two and three molecular order parameters, respectively. Some counter-examples indicate that these points are not general, and in some cases ambiguities are present in their analysis. The role of conformational changes cannot be neglected.
\end{abstract}

The use of deuteron magnetic resonance (DMR) to study the molecular dynamics of liquid crystals has increased during recent years [1-7]. The method is very specific : DMR spectra of deuterated liquid crystals exhibit well-resolved quadrupolar doublets which characterize the motional averaged orientation of each C-D bond in the molecule. With the flexible mesogens studied until now, two contributions to the motional averaging are anticipated : molecular conformational changes and motion of the molecule as a whole. In general the number of orientational order parameters required is considerably greater than the number of independent quadrupolar splittings which can be measured. In order to simplify the quantitative interpretation, a currently used approximation is to describe the molecular orientational order relative to the director by a single order tensor $\mathbf{S}$ common to the entire mesogen. Then any splitting $\delta v_{i}$ of a deuteron $\mathrm{D}_{i}$ may be simply expressed in terms of the different components $A, B, \ldots$ of this tensor [8] :

$$
\delta v_{i}(T)=a_{i}(T) A(T)+b_{i}(T) B(T)+\cdots
$$

$\left(^{*}\right)$ Part of this work has been presented at the Seventh International Liquid Crystals Conference (Bordeaux 1978).

$\left.{ }^{* *}\right)$ Laboratoire associé au C.N.R.S. where $a_{i}(T), b_{i}(T), \ldots$, are averages over the conformational changes. If there are $N$ independent molecular order parameters, then they may be extracted from the splittings of $N+1$ different sites so that one splitting may be connected to $N$ others by a linear relationship. When only two order parameters are required, this linear equation may be written :

$$
\delta v_{i}(T)=c_{j}(T) \delta v_{j}(T)+c_{k}(T) \delta v_{k}(T)
$$

where the conformation constants $c_{j}$ and $c_{k}$ depend on the conformational averages, the $a$ 's and $b$ 's. Following such an approach, Bos and Doane [9] recently concluded that the conformation constants of complex mesogens are independent of temperature. They found that ratio plots, such as $\delta v_{i} / \delta v_{k}$ vs. $\delta v_{j} / \delta v_{k}$, are always straight lines in a system having nematic, smectic A and smectic B phases (so that the temperature dependence of one splitting can be determined from two others). Furthermore a discontinuity in the corresponding linear rátio plot at a nematic-smectic $\mathrm{C}$ transition was interpreted only as the manifestation of the need to introduce a third molecular order parameter. This procedure allowed the authors to draw conclusions about the invariance of the average conformation of the molecule throughout the entire mesomorphic range and the necessity of using two 
or three order parameters for uniaxial or biaxial (smectic C) mesophases. One may ask if this simple description is reliable and general, irrespective of the nature of the mesogen. In this paper we present a detailed analysis of the behaviour of different ratio plots which conflicts with the Bos and Doane interpretation. We emphasize the complexity of dynamical process underlying the DMR data of currently studied mesogens.

The proposed correspondence [9] between the symmetry of the mesophase and the number of order parameters required to specify the splittings $\delta v_{i}(T)$ is not general. This is clearly illustrated in figure 1 where we have made a ratio plot $\left(\delta v_{2} / \delta v_{4}\right.$ vs. $\left.\delta v_{3} / \delta v_{4}\right)$, similar to the one of Ref. [9], using the data on terephthalidene-di-p- $\mathrm{d}_{9}$-butyl-2,6- $\mathrm{d}_{2}$-aniline or TBBA$d_{22}$ [2]. The plot can be approximated as a straight line in the nematic phase. However, a discontinuity, well outside of the error limits of the data, appears at the nematic-smectic A transition (similar in magnitude to the one observed in Ref. [9] at the nematicsmectic C transition of octyloxybenzoic acid [10]). On the other hand, the plot is continuous at the smectic A-smectic C transition. Then, the implied interpretation is that the number of order parameters

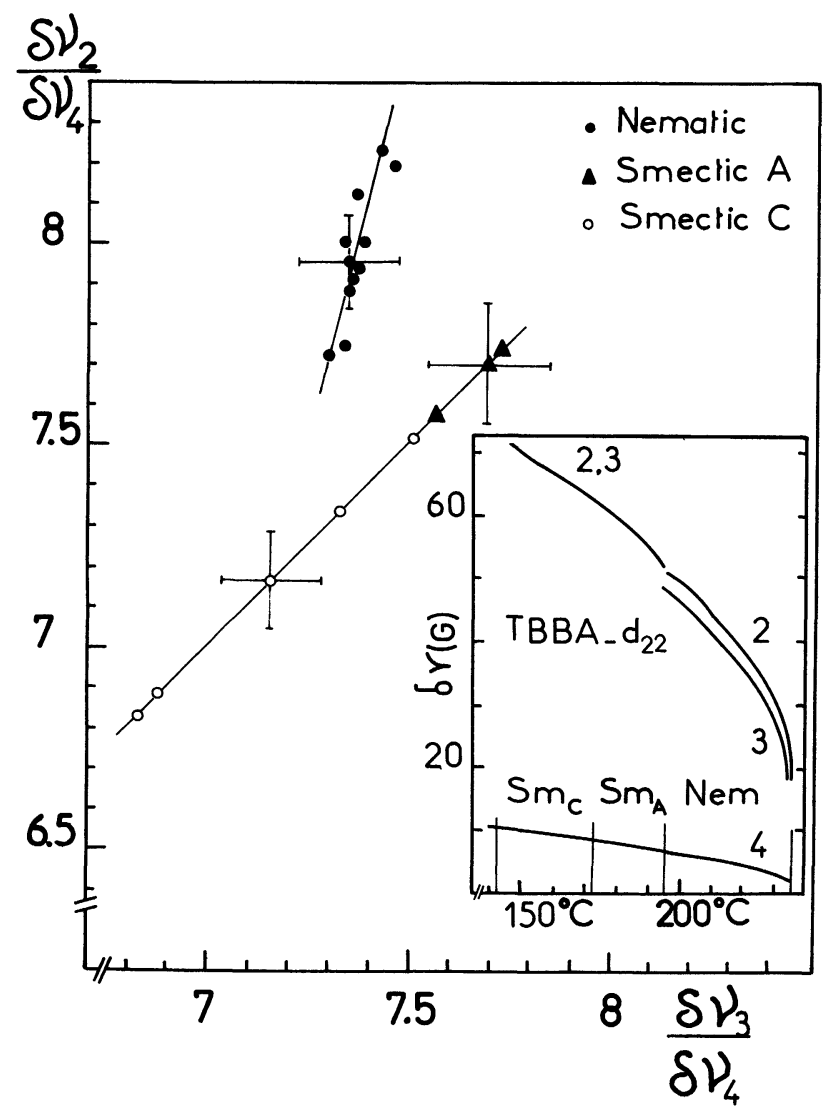

Fig. 1. - Plot of ratios of deuteron splittings obtained at different temperatures for TBBA- $\mathrm{d}_{22}$. The indices of the splittings are associated with the position of the chain-segment. The inset shows a sketch of the data (Ref. [2]) from which the splittings were obtained. The magnitude of the indicated error limits results from a statistical analysis of multiple spectra within the same mesophase.

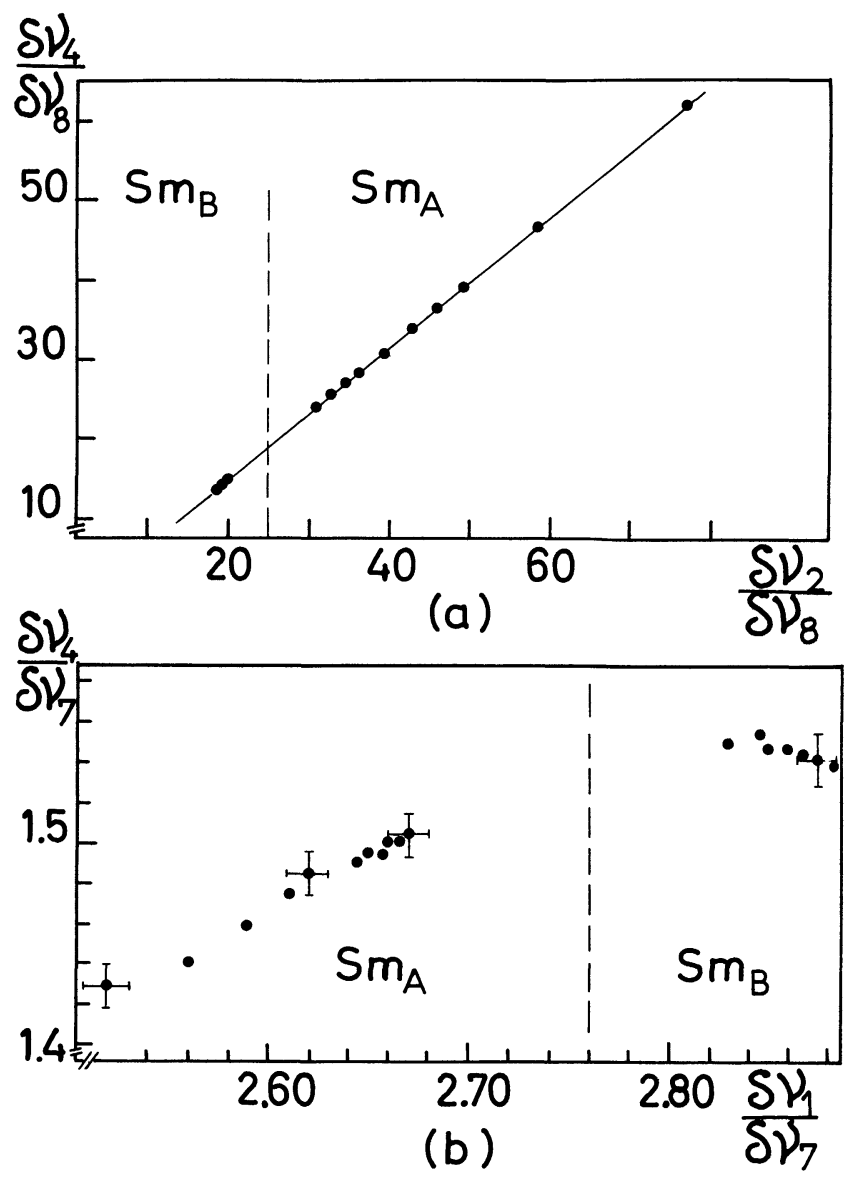

Fig. $2 a$ ) and $b$ ). - Two different ratio plots using splittings obtained from the compound BOBOA- $d_{19}$ at various temperatures of the uniaxial smectic $A$ and $B$ phases. The indices of the splittings are associated with the position of the chain-segment.

must be modified at the transition involving no phase symmetry change, whereas it remains constant at the transition involving a symmetry change.

The number of order parameters deduced is not independent of the set of splittings $\delta v_{i}$ used in the ratio plots. This is illustrated in figure 2 where we have studied the uniaxial smectic A and B phases of p-butyloxybenzylidene-p$\mathrm{d}_{17}$-octyl-2,6- $\mathrm{d}_{2}$-aniline (BOBOA- $\mathrm{d}_{19}$ ) [11] using different $\delta v_{i}$ in the ratios. Contrasting figures $2 a$ and $2 b$ indicate that the Bos and Doane approach does not yield consistent results. Consider the smectic B phase, for example. Two order parameters are sufficient according to figure $2 a$ (the conclusion in Ref. [9]), whereas a third one is necessary according to figure $2 b$. This discrepancy reflects the disparate sensitivity of the ratio plots to the existence of multiple order parameters.

The concept that the average molecular conformation remains unchanged at phase transitions is not corroborated in some direct observations. Consider the DMR data of TBBA- $\mathrm{d}_{22}$, for example. As indicated in Ref. [2] (see also the inset of figure 1), the deuteron splittings of the second and third segment of 
the chain remain identical throughout the smectic $\mathrm{A}$ range $\left(\delta v_{2}(T)=\delta v_{3}(T)\right)$; on heating the system into the nematic phase, two quadrupolar doublets are observed $\left(\delta v_{2}(T) \neq \delta v_{3}(T)\right)$. Thus, unless one modifies the number of order parameters, the appearance of a second doublet requires significant changes in the molecular conformational averages at this smectic Anematic transition. For this particular case, conformational changes may be correlated with the transition between translationally ordered and disordered structures. The same type of argument may also be used for other mesophase transitions where similar DMR observations have been reported [7] [11].

These difficulties lead us to a critical analysis of the linearity of ratio plots such as the one for BOBOA presented in figure 1 of Ref. [9], i.e.

$$
\delta v_{7} / \delta v_{8}=c_{2} \delta v_{2} / \delta v_{8}+c_{8} \text {. }
$$

Following the Bos and Doane analysis, we look for the best straight line fitting the ratio of the observed splittings by means of a linear least-square regression over the entire mesomorphic range. Such a straight line is found to be characterized by the constants

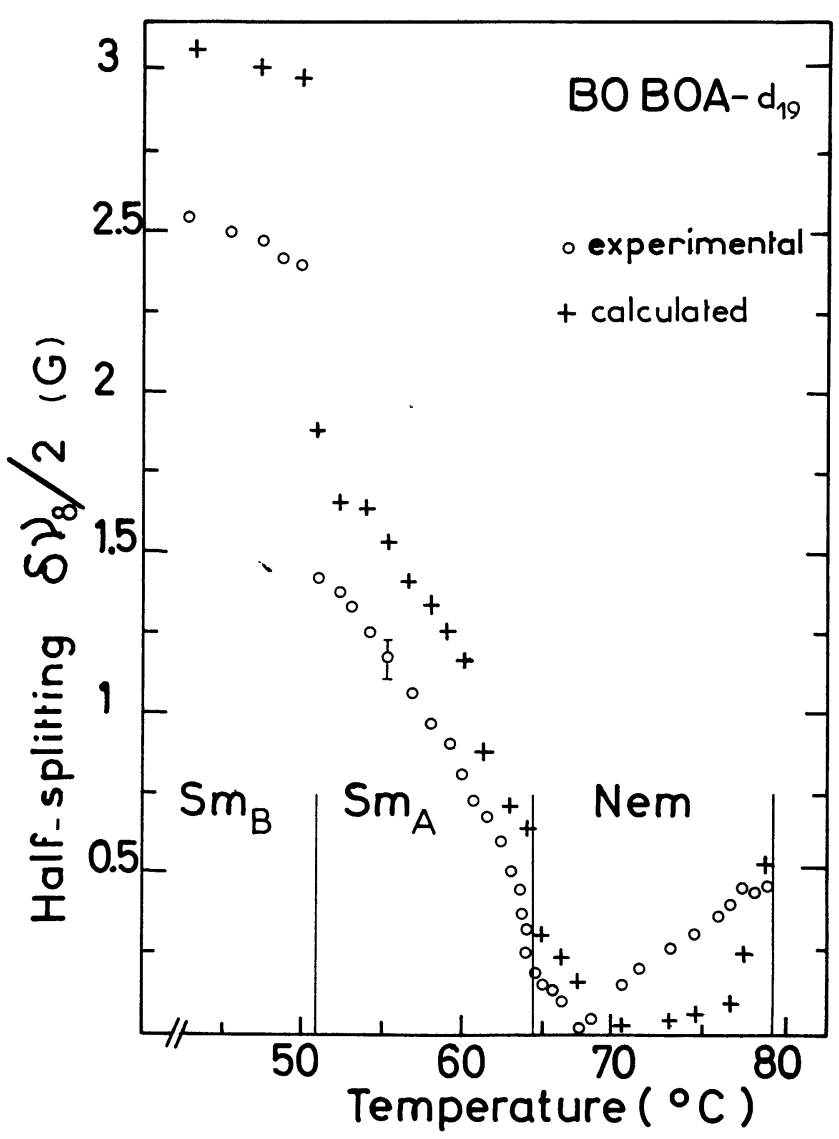

Fig. 3. - Comparison of the experimental and calculated variations of the methyl splitting $\delta v_{8}(T)$ of the compound BOBOA-d ${ }_{19}$. The calculated data were obtained by means of the typical relation connecting $\delta v_{8}(T)$ to $\delta v_{2}(T)$ and $\delta v_{7}(T)$ when the splittings variations are assumed to be governed by two order parameters. The calculation procedure is detailed in the text. The characteristic spectral linewidth is indicated in the Sm A phase by the vertical bar. $\bar{c}_{2}=0.582$ and $\bar{c}_{8}=-1.693$. Then the obvious test of the reliability of this linear fit is, going the other way round, to calculate the temperature dependence of one of the splittings using the experimental values of the two others. We attempt this process in figure 3 for the methyl splitting $\delta v_{8}(T)$ which presents a very unusual temperature dependence [11]. Although the features of the experimental curve are reproduced, it appears that the calculation does not fit in detail all the experimental data : the observed discrepancies, much larger than the spectral linewidth, indicate the determined constants $\bar{c}_{2}$ and $\bar{c}_{8}$ differ from the actual conformational coefficients $c_{2}(T)$ and $c_{8}(T)$ which connect the considered ratios in a given temperature range. Moreover, if the two constants are adjusted to get a best fit locally (in the temperature range of any one mesophase) the disparity between calculated and observed $\delta v_{8}(T)$ persist in other mesophases. The irreversibility of such a procedure shows that this type of analysis can only be considered as a rough approximation of the real phenomena. In fact the lack of sensitivity of the plot presented by Bos and Doane to the variations of the $c$ 's arises mainly from the use of the very small splittings $\delta v_{8}$ [11] in the denominator of the ratios, yielding extremely expanded scales. Figure 3 demonstrates that the ratio plots cannot be used to exclude changes in the average molecular conformation with temperature in a quantitative analysis of $\delta v_{i}(T)$.

A precise interpretation of the present DMR data on thermotropic systems is not trivial. Since the order tensor $\mathbf{S}$ is symmetric and traceless, the splittings for a mesogen molecule of low symmetry should depend in principle on five independent, non zero orientational order parameters, irrespective of the symmetry, uniaxial or biaxial, of the mesophase (provided that the magnetic field is kept along the direction of the mean molecular alignment [12]). Molecular motions more rapid than the time scale of DMR experiments may effectively increase the symmetry of the molecule so that the number of required order parameters is reduced. Under these conditions any change in this number through the mesomorphic range is necessarily related to a change in the average molecular symmetry. So it seems reasonable to anticipate that the number and value of additional order parameters in a given mesophase might be different for mesogens from quite different chemical structural classes. The corresponding effects on the splitting $\delta v_{i}(T)$ always appear inextricably convoluted with conformational terms $\left(a_{i}(T), b_{i}(T) \ldots\right.$ in Eq. (1)). In view of these complicating factors, one simple way to interpret qualitatively the changes in $\delta v_{i}(T)$ is to use only the principal order parameter, $S_{z z}$, and to consider that the conformational averages at different molecular sites are temperature dependent [11]. Such a description may be justified in first approximation by the small magnitudes anticipated for additional order parameters. In the nematic phase, for example, the asymmetry coefficient 
$\Delta S=S_{x x}-S_{y y}$ which has been measured [13] and estimated [14] is very small compared to $S_{z z}$ [15]. In a single order parameter approach, the particularities observed in the $\delta v_{i}(T)$ at phase transitions can be attributed in part to abrupt changes in the value of $S_{z z}$ (related to the thermodynamic nature of the phase transition [1]) and to changes in the average molecular conformation (related to changes in the average orientation of the long axis relative to a given C-D bond [11]). These conformational changes, which may take place in the aromatic part as well as in the aliphatic part of the mesogen are corroborated by other techniques. For example, Raman spectroscopy shows that the statistical weight of the trans and gauche states in the end-chains varies within the nematic phase [16]. In addition volumetric measurements indicate that the mean lateral area per molecule increases throughout the smectic range [17]. Corresponding changes in the mesogen average conformation would be expected.

The existence of several order parameters cannot be ignored even in uniaxial mesophases. From the examples quoted above it is clear that the use of ratio plots does not allow one to determine reliably the exact number and the magnitude of the order parameters. A thorough analysis necessarily implies the investigation of multiple interactions on the same chemical group or rigid segment of the mesogen by means of more sophisticated techniques such as the ones recently developed in Ref. [13] and [18].

The authors are very grateful to Dr. E. T. Samulski (Storrs, Connecticut) and to Professor M. Bloom (U.B.C., Vancouver) for stimulating discussions and a critical reading of the manuscript.

\section{References}

[1] Luz, Z., Hewitt, R. C. and Мeiвoом, S., J. Chem. Phys. 61 (1974) 1758

[2] Deloche, B., Charvolin, J., Liebert, L. and Strzelecki, S., J. Physique Colloq. 36 (1975) C1-21.

[3] Emsley, J. W., Lindon, J. C. and Luckhurst, G. R., Mol. Phys. 30 (1975) 1913.

[4] Diehl, P. and Tracey, A. S., Mol. Phys. 30 (1975) 1917.

[5] Bos, P. J., Pirs, J., Ukleja, P., Doane, J. W. and Meubert, M. E., Mol. Cryst. and Liq. Cryst. 40 (1977) 59.

[6] Dong, R. Y., Tombuck, E., Wade, C. E., Visintainer, J. J. and Bock, E., J. Chem. Phys. 66 (1977) 4121.

[7] Hsi, S., Zimmerman, H. and LuZ, Z., J. Chem. Phys. 69 (1978) 4126.

[8] If a molecular axes system $(x, y, z)$ can be defined, these terms $A, B, C \ldots$ have to be identified with the components $S_{\mu \nu}$ $(\mu, v=x, y, z)$ of the order tensor $\mathbf{S}$ defined by A. Saupe SAUPE, A., Naturforsh 19 a (1964) 161 .

[9] Bos, P. J. and Doane, J. W., Phys. Rev. Lett. 40 (1978) 1030.

[10] The use of dimerized carboxylic acids to study such subtle aspects of the smectic-nematic transition should be questioned ; indeed it is known that the lifetime of these hydrogen bonded configurations in the nematic phase is controlled by orientational motions of the molecules
(Deloche, B. and Cabane, B., Mol. Cryst. Liq. Cryst. 19 (1972) 25). This association-dissociation process may be profoundly affected at phase transitions and may in turn, modify the average molecular conformation.

[11] Deloche, B. and Charvolin, J., J. Physique 37 (1976) 1497. DelOCHE, B., Thesis, University of Paris-XI, Orsay (1978).

[12] Luz, Z. and Меiвоом, S., J. Chem. Phys. 59 (1973) 275. (See appendix).

[13] Emsley, J. W., LuCKhuRst, G. R., Gray, G. W. and Mosley, A., Mol. Phys. 35 (1978) 1499.

[14] Volino, F. and Dianoux, A. J., Mol. Cryst. Liq. Cryst. Lett. 49 (1979) 153.

[15] In fact there is one order of magnitude discrepancy between the values of $\Delta S$ deduced from ratio plots (P. J. Bos and J. W. Doane : communication presented at the Seventh Liquid Crystals Conference, Bordeaux 1978 ; to be published) and that measured [13].

[16] Destrade, C., Gullon, F. and Gasparoux, H., Mol. Cryst. Liq. Cryst. 36 (1976) 115.

[17] Guillon, D. and Sxoulios, A., J. Physique 38 (1977) 79.

[18] Higgs, T. P. and MacKay, A. L., Chem. Phys. Lipids 20 (1977) 105. 